\title{
Monodispersed Bioactive Glass Nanoclusters with Ultralarge Pores and Intrinsic Exceptionally High miRNA Loading for Efficiently Enhancing Bone Regeneration
}

\author{
Yumeng Xue, Yi Guo, Meng Yu, Min Wang, Peter X. Ma, and Bo Lei*
}

Bioactive glass nanoparticles (BGNs) have attracted much attention in drug delivery and bone tissue regeneration, due to the advantages including biodegradation, high bone-bonding bioactivity, and facile large-scale fabrication. However, the wide biomedical applications of BGNs such as efficient gene delivery are limited due to their poor pore structure and easy aggregation. Herein, for the first time, this study reports novel monodispersed bioactive glass nanoclusters (BCNCs) with ultralarge mesopores (10-30 nm) and excellent miRNA delivery for accelerating critical-sized bone regeneration. BGNCs with different size $(100-500 \mathrm{~nm})$ are fabricated by using a branched polyethylenimine as the structure director and catalyst. BGNCs show an excellent apatite-forming ability and high biocompatibility. Importantly, BGNCs demonstrate an almost 19 times higher miRNA loading than those of conventional BGNs. Additionally, BGNCs-miRNA nanocomplexes exhibit a significantly high antienzymolysis, enhance cellular uptake and miRNA transfection efficiency, overpassing BCNs and commercial Lipofectamine 3000. BCNCs-mediated miRNA delivery significantly improves the osteogenic differentiation of bone marrow stromal stem cells in vitro and efficiently enhances bone formation in vivo. BGNCs can be a highly efficient nonviral vector for various gene therapy applications. The study may provide a novel strategy to develop highly gene-activated bioactive nanomaterials for simultaneous tissue regeneration and disease therapy.

life. ${ }^{[1]}$ Although bone tissue possesses the self-repair ability, the large critical-size bone defect cannot regenerate without bone grafting and $5-10 \%$ fractures show nonunion or delayed healing. ${ }^{[2]}$ As the noncoding RNAs $(\approx 22 \mathrm{nt})$, microRNAs (miRNAs) can regulate the fundamental physiological process in various organisms through degrading and repressing the expressions of target RNAs. ${ }^{[3]}$ In recent years, various miRNAs have shown the capacity to positively regulate the osteogenic genes expressions and osteogenesis in vitro and in vivo through multiple signaling pathways. ${ }^{[4]}$ Unfortunately, naked miRNAs are difficult to enter cells and also easy to be degraded by the nuclease under physiological environment. Different gene vectors have been synthesized and used to deliver miRNA for enhanced bone tissue formation. ${ }^{[5]}$

Viral vectors exhibited good miRNA transfection efficiency but also showed some negative effects such as infection and immunogenicity. ${ }^{[6]}$ To avoid the problems of viral vectors, nonviral vectors

\section{Introduction}

The traumas and diseases resulted bone tissue loss has become huge social problems seriously affecting the human quality of

Dr. Y. Xue, Dr. Y. Guo, Dr. M. Yu, Dr. M. Wang, Prof. B. Lei

Frontier Institute of Science and Technology

Xi'an Jiaotong University

Xi'an 710049, China

E-mail: rayboo@xjtu.edu.cn, leiboaray@foxmail.com

Homepage: http://gr.xjtu.edu.cn/web/rayboo

Prof. P. X. Ma

Department of Biologic and Materials Sciences

University of Michigan

Ann Arbor, MI 48109-1078, USA

Prof. B. Lei

State Key Laboratory for Mechanical Behavior of Materials

Xi'an, Jiaotong University

Xi'an 710049, China

Prof. B. Lei

State Key Laboratory for Manufacturing Systems Engineering

Xi'an Jiaotong University

Xi'an 710054, China

DOI: $10.1002 / \mathrm{adhm} .201700630$ including cationic polymers and liposomes were widely investigated for miRNA delivery in vitro and in vivo. ${ }^{[7-9]}$ However, the miRNA delivery of cationic polymers and liposomes was always unsatisfactory due to the cytotoxicity, low transfection efficiency, and serum stability. ${ }^{[10]}$ It is necessary to develop next generation nonviral vectors with good osteogenic biocompatibility, high miRNA loading and protecting ability, and excellent miRNA delivery efficiency.

As compared to conventional inorganic biomaterials, bioactive glasses (BGs) have inspired intensive research interest in bone tissue regeneration due to their remarkable osteoblast biocompatibility, bioactivity including the chemical bone-bonding ability and gene activation properties. ${ }^{[11-16]}$ Therefore, BG nanoparticles (BGNs) have a great potential to be employed as novel bioactive nonviral gene vectors for tissue regeneration and gene therapy. Up to now, few studies reported the gene delivery applications based on BGNs. For instance, Kim used aminefunctionalized mesoporous BGNs to deliver small interfere RNA (siRNA) and 5.18\% loading efficiency was obtained. ${ }^{[17]}$ Chen and co-workers developed an amine-modified radial mesoporous BGNs which showed good DNA delivery efficiency and protection ability. ${ }^{[18]}$ Although some positive results of 
BGNs in DNA and siRNA delivery were achieved, the aminefunctionalization was necessary to make BGNs positively charged for loading genes. The limited surface positive charge density usually resulted in the low loading and poor transfection efficiency. Additionally, the charged surface of nanoparticles may also cause the nonspecific protein adsorption and cytotoxicity in vivo applications. ${ }^{[19]}$

Recently, our group demonstrated that BGNs could bind miRNA directly without any surface cationic modification and release them in cellular environment, suggesting the great potential of BGNs as the next generation nonviral vectors for enhanced gene therapy. ${ }^{[20]}$ Through the strong interaction between calcium in BGNs and phosphates in miRNA, BGNs showed a significantly high miRNA loading and intracellular transfection efficiency, as compared to conventional silica nanoparticles. ${ }^{[20]}$ Even so, as an ideal gene vector, the small mesopore size $(<5 \mathrm{~nm})$ and pore volume $\left(<0.04 \mathrm{~m}^{3} \mathrm{~g}^{-1}\right)$ of BGNs was still not satisfied in biomedical applications. ${ }^{[21,22]}$ It is very worthwhile to develop BGNs with the novel nanostructure for enhanced gene delivery applications.

Branched polyethyleneimine (PEI) is a common functional macromolecule that usually used for gene delivery, but is rarely employed as template to direct the formation of nanostructure. ${ }^{[23]}$ Based on the fact that the abundant amino in PEI can catalyze the hydrolysis of silica precursor and the branched structure may direct the formation of large pores, here, for the first time, we developed monodispersed bioactive glass nanoclusters (BGNCs) with ultralarge pores and controlled size. We also demonstrated their highly efficient miRNA delivery, activated osteogenic genes expression in vitro and enhanced critical-sized bone defect regeneration in vivo, as compared to conventional BGNs.

\section{Results and Discussion}

\subsection{Preparation and Characterization of Monodispersed BGNCs}

In this work, we have developed a facile and simple method to fabricate the monodispersed BGNCs by employing a branched macromolecules PEI as structure director and reaction catalyst. The synthesize process, reaction mechanism, and potential miRNA delivery application is illustrated in Figure 1. On one hand, the amine-based polymer PEI could serve as an in situ catalyst for silica precursor hydrolysis and condensation. On the other hand, PEI could self-assemble into the framework during condensation reactions, therefore act as a pore expansion agent to generate ultralarge pores (Figure 1A). Based on the strong gene binding ability and ultralarge pores, BGNCs could serve as a high efficient nonviral vector

A
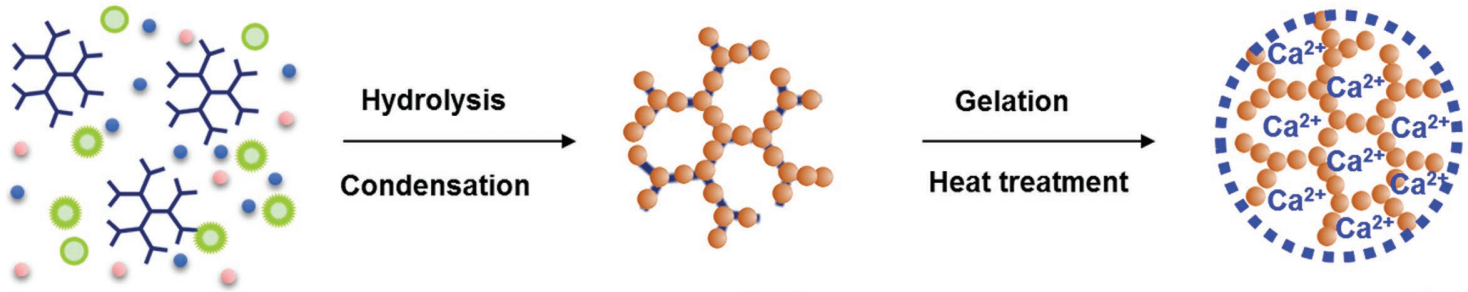

B
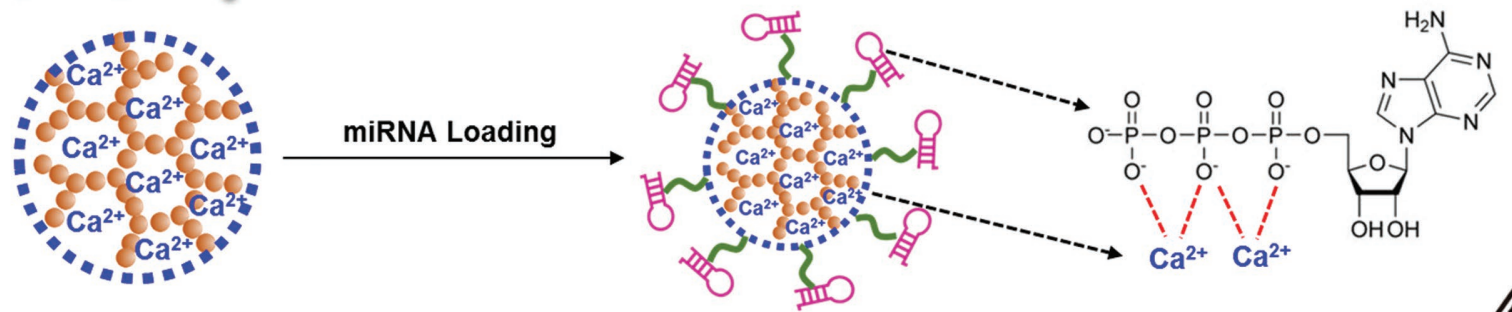

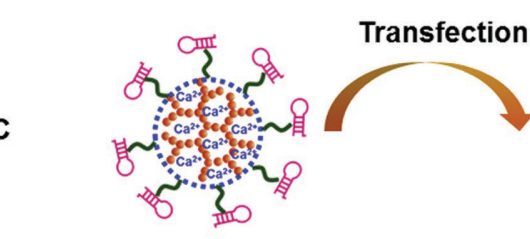

BGNC@miRNA

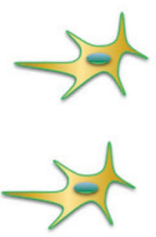

BMSCs

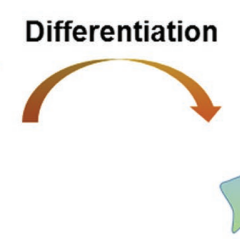

Osteoblasts

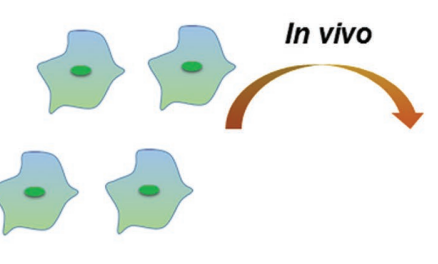

Rapid bone regeneration

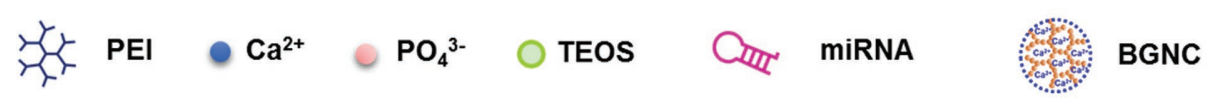

Figure 1. Schematic illustration showing the fabrication and miRNA delivery application of BGNCs. A) Synthesis of BGNCs by a branched PEI-assistant sol-gel process. B) Ultrahigh miRNA loading ability and binding mechanism. C) BGNC-based miRNA delivery to regulate osteogenic activity of stem cells in vitro and bone regeneration in vivo. 
to deliver miRNA for accelerating bone tissue regeneration (Figure 1B,C).

The final nanostructure and size of the synthesized BGNCs could be tuned by the concentration of PEI during the reaction. As shown in Figure 2A-D, BGNCs showed a typical nanoclusters morphology and large mesopores. Specifically, the morphology and size of BGNCs could be effectively tailored by the mass ratio of PEI. 10\%BGNCs, $20 \%$ BGNCs, and $30 \%$ BGNCs exhibited a size around 100, 200, and $450 \mathrm{~nm}$, respectively (Figure S1, Supporting Information). Generally, the monodispersed size of BGNCs was increased from 100 to $450 \mathrm{~nm}$ with the increase of PEI contents. However, when the mass ratio of PEI decreased to $6 \%$, BGNCs presented a nonuniform size with a wide range from 50 to $500 \mathrm{~nm}$ (Figure S2, Supporting Information). Under the high concentration of PEI, the rapid hydrolysis and condensation of silanol groups took place, leading to the fast formation of silica network. Then the smaller nanoclusters would aggregate and produce a larger one after further reaction. However, at the low concentration of PEI, the incomplete hydrolysis and condensation of silanol groups would result in the nonuniform size and irregular morphologies of BGNCs. Through varying the amount of PEI, the size, microstructure and morphology of BGNCs could be controlled facilely. In particular, through increasing the amount of precursors and solvents, the large-scale production of BGNCs can be achieved, and their morphology/size distribution were almost unchanged (Figure S3, Supporting Information).

BGNCs exhibit a strong and broad band at $2900-3500 \mathrm{~cm}^{-1}$ and $1600 \mathrm{~cm}^{-1}$ which were attributed to the stretching and bending vibration of hydroxyls (Figure 2E). The characteristic peaks of BGNCs can be found at 1060, 800 , and $480 \mathrm{~cm}^{-1}$. [24] The strong absorbance at $1060 \mathrm{~cm}^{-1}$ is assigned to the stretch vibration of Si-O-Si, while the peaks at 800 and $480 \mathrm{~cm}^{-1}$ were owing to the bending vibration of Si-O-Si. The broad peak in
XRD patterns indicated the typical amorphous structure of BGNCs (Figure 2F). The energy dispersive spectroscopy (EDS) analysis confirmed the chemical composition of BGNC containing Si and Ca (Figure 2G). To investigate the pore structure and property of BGNCs, the specific surface area, pore volume, and pore size distribution of BGNCs and BGNs were determined (Figure 2H; and Figure S4, Supporting Information). The Brunauer-Emmet-Teller (BET) surface area of 10\%BGNCs and BGNs are calculated to be 213.611 and $236.620 \mathrm{~m}^{2} \mathrm{~g}^{-1}$, respectively (Table S2, Supporting Information). The 10\%BGNC showed a total pore volume of $1.138 \mathrm{~cm}^{3} \mathrm{~g}^{-1}$ and average pore size of $20.056 \mathrm{~nm}$, which were almost 30 and 6 times higher than those of BGNs $\left(0.0342 \mathrm{~cm}^{3} \mathrm{~g}^{-1}\right.$ and $3.468 \mathrm{~nm}$ ) (Table S2, Supporting Information). The ultralarge porous structure may endow BGNCs the high gene storage and delivery ability for efficient bone tissue regeneration.

\subsection{Apatite-Forming Evaluation of BGNCs}

BGs have been successfully exploited as a kind of highly bioactive material for bone regeneration because of its improved apatite-forming ability as compared to other conventional bioactive ceramics. The well-formed apatite layer on BGs in physiological environment can bond to bone and even stimulate new bone growth. ${ }^{[25,26]}$ In this work, the apatite-forming ability of the synthesized BGNCs was studied in detail. After soaking in simulated body fluids (SBF) for $3 \mathrm{~d}$, the flake-like nanocrystals were observed clearly on BGNCs (Figure 3A). Further Fourier transform infrared (FTIR) spectra indicated the presence of three new peaks at 550,605 , and $950 \mathrm{~cm}^{-1}$ which are associated to the vibration of crystalline P-O in crystalline $\mathrm{PO}_{4}{ }^{3-}$ (Figure 3B). ${ }^{[27]}$ The characteristic peaks of hydroxyapatite nanocrystals (JCPDS 09-432) could also be observed from the
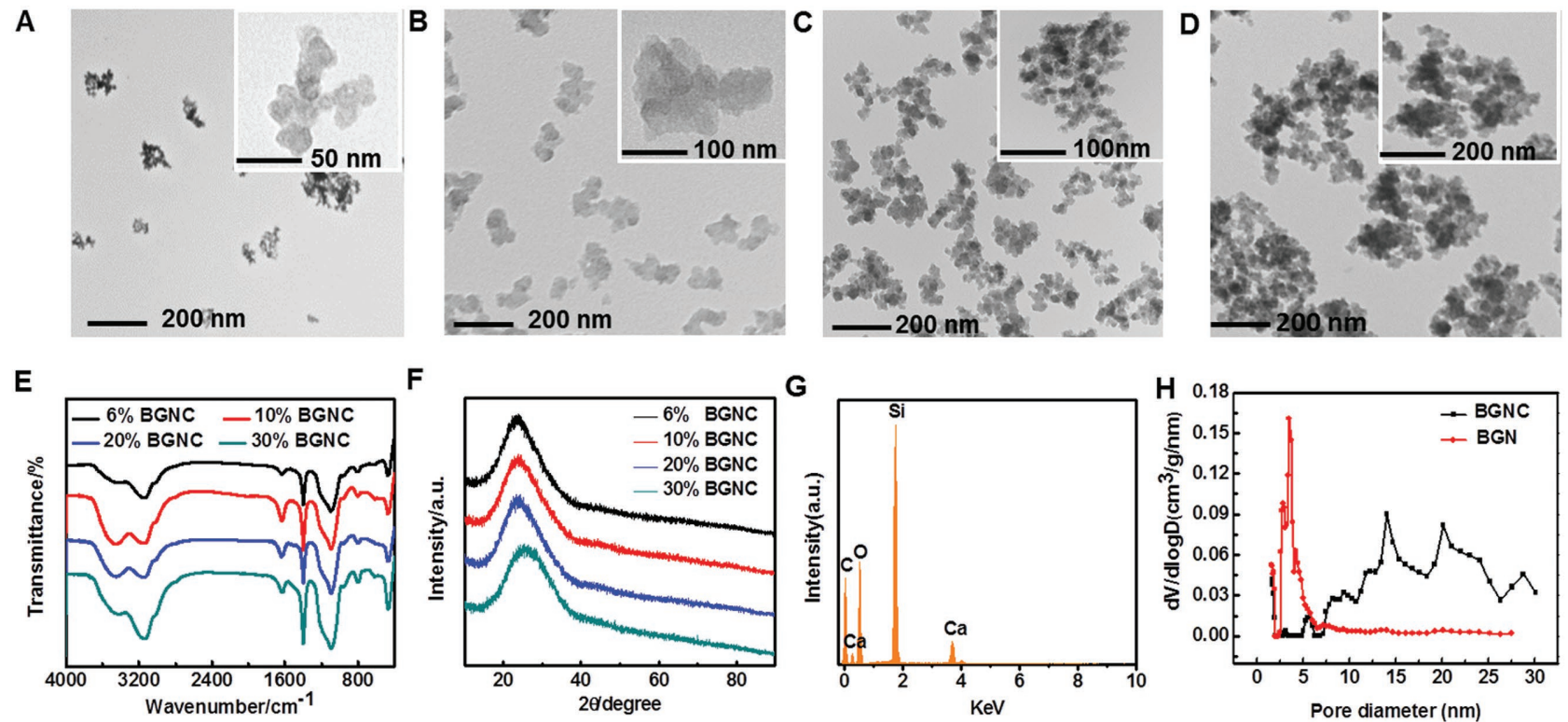

Figure 2. Structure and characterizations of BGNCs. TEM images showing the monodispersed cluster-like morphology and nanoscale size of $A$ ) $6 \%$ BGNC, B) $10 \%$ BGNC, C) $20 \%$ BGNC, D) $30 \%$ BGNC. E) FTIR spectra exhibiting chemical structure of BGNCs. F) XRD patterns presenting the typical amorphous structure of BGNCs. G) EDS analysis identifying the element composition of BGNCs. H) Pore size distributions of BGNs and BGNCs based on $\mathrm{N}_{2}$ adsorption-desorption analysis. 
A
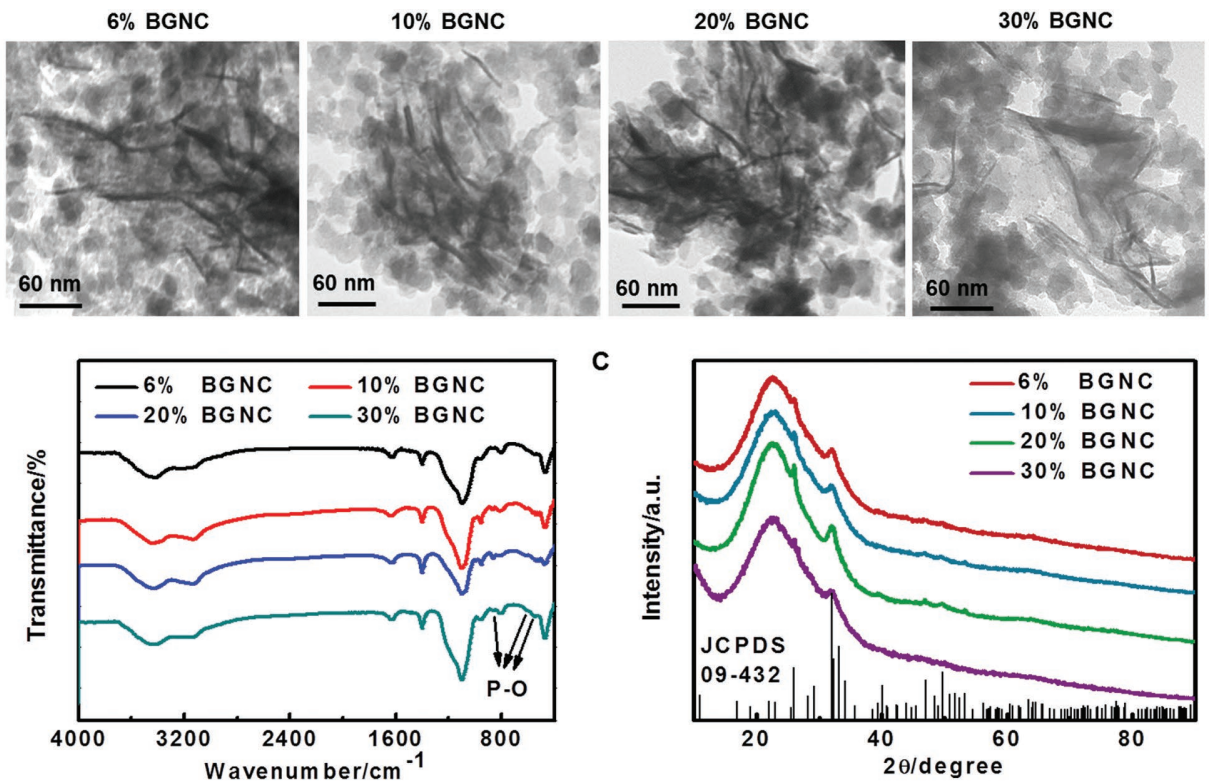

Figure 3. Apatite-forming ability evaluation of BGNCs after soaking in SBF for $3 \mathrm{~d}$. A) TEM image showing the apatite nanocrystals formed on surface of BGNCs. B) FTIR spectra indicating the chemical structure of new formed hydroxyapatite nanocrystals on BGNCs. C) XRD patterns exhibiting the characteristic peaks of hydroxyapatite nanocrystals on BGNCs (JCPDS No 09-432).

XRD pattern at $26^{\circ}(002), 32^{\circ}(211)$, and $46^{\circ}(222)$, which further implied the formation of hydroxyapatite layer (Figure 3C). ${ }^{[27]} \mathrm{By}$ investigating the bioactivity of BGNC utilizing FTIR, XRD, and TEM, we can reasonably conclude that the obtained BGNCs possess excellent apatite-forming ability, and the superior bioactivity make it can be widely used in bone tissue repair and regeneration.

\subsection{Ultrahigh miRNA Loading and Stability of BGNCs}

Compared with BGNs and conventional mesoporous materials, BGNCs possess ultralarge mesopores and may have high miRNA loading ability. Figure 4A-C reveals the miRNA loading

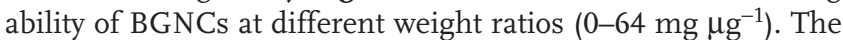
miRNA was retarded completely by BGNCs at a weight ratio of
A

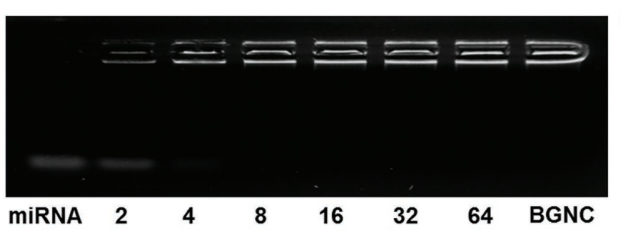

B

D

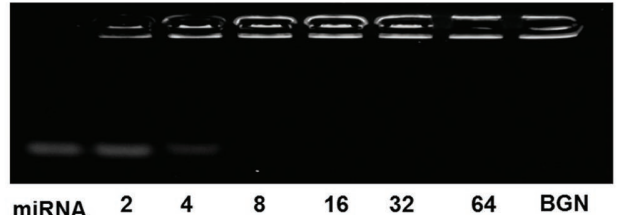

Naked miRNA

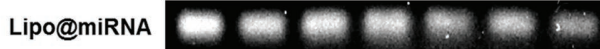

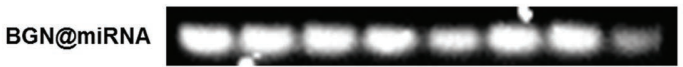

BGNC@miRNA

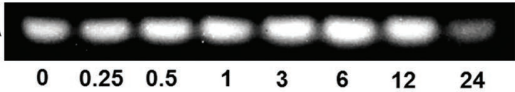

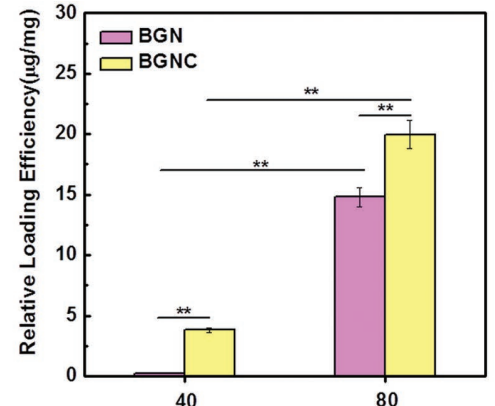

E

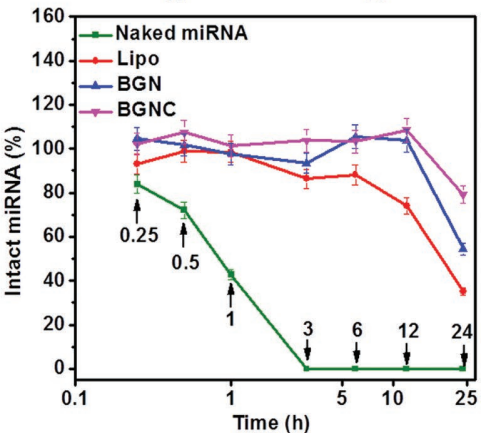

Figure 4. Ultrahigh miRNA loading and protection capacity of BGNCs. Gel retardation pictures showing the miRNA binding ability of A) BGNCs and B) BGNs with different weight ratios to miRNA (2-64 mg $\left.\mathrm{mg}^{-1}\right)$. C) Quantitative miRNA loading efficiency of BGNC and BGN at 40 and $80 \mu \mathrm{g} \mathrm{mL}$ $(* x p<0.01, n=5)$. D) Antinuclease degradation ability of various miRNA complexes after soaking in serum solution for $24 \mathrm{~h}$. E) Quantitative miRNA intact percentage of different samples. 
4:1 (Figure 4A), overpassing that of BGNs (8:1) (Figure 4B). The quantitative analysis showed that BGNCs demonstrated significantly high miRNA loading efficiency $\left(3.8 \mu \mathrm{g} \mathrm{mg}^{-1}\right)$ which was almost 19 times high compared to BGNs $\left(0.2 \mu \mathrm{g} \mathrm{mg}^{-1}\right)$ at the same nanoparticles concentration $\left(40 \mu \mathrm{g} \mathrm{mL}^{-1}\right)$. The high loading ability of BGNCs was probably due to two reasons. On one hand, the $\mathrm{Ca}^{2+}$ in BGs framework has a strong binding affinity to the phosphates groups in miRNA structure, which contributed to their loading ability. ${ }^{[28]}$ This strong gene binding mechanism of BGs through a metal ion complex process has been demonstrated by our previous report. ${ }^{[20]}$ In addition to the calcium-miRNA complex interaction, the ultralarge porous structure of BGNCs should also contribute to their high miRNA loading amount. BGNCs have a 30 times and 6 times high pore volume and mean pore size comparing with BGNs, thus showed the ultrahigh miRNA loading efficiency. The large pore volume and size derived high gene or drug loading performance was reported everywhere, especially for mesoporous inorganic nanomaterials. ${ }^{[2,30]}$ The antinuclease degradation ability of miRNA complexes (BGNC@miRNA, BGN@miRNA) was investigated by incubating them with serum, Lipo, and naked miRNA were chose as controls (Figure 4D,E). It can be found that both BGNCs and BGNs could protect the miRNA from the enzymolysis by nuclease within $24 \mathrm{~h}$, and almost no significant degradation of miRNA could be detected (Figure 4D). Naked miRNA was degraded completely after $3 \mathrm{~h}$ (Figure 4E). However, after $24 \mathrm{~h}$ incubation, more than $80 \%$ of the intact miRNA could be detected in BGNC@miRNA group, but only $50 \%$ and $35 \%$ of intact miRNA could be detected in BGN@ miRNA and Lipo@miRNA group, suggesting the excellent miRNA protection ability of BGNCs (Figure 4E). The excellent
miRNA protecting ability of BGNC was mainly owing to the large pore size and volume of BGNCs. On one hand, the large pores created more chances for the interaction between $\mathrm{Ca}^{2+}$ and the guest genes, therefore significantly enhanced the binding forces to miRNA and avoided the fast release of miRNA. On the other hand, the large pore volume of BGNCs could effectively protect miRNA from enzymolysis. The ultrahigh miRNA loading and excellent antinuclease degradation ability of BGNCs enabled their promising application in gene delivery.

\subsection{In Vitro Cell Biocompatibility, miRNA Transfection, and Osteogenic Differentiation}

The bone marrow stromal stem cells (BMSCs) viability and proliferation behavior was investigated through incubating with BGNCs at different concentrations $\left(40-160 \mu \mathrm{g} \mathrm{mL} \mathrm{m}^{-1}\right)$. After culture with BGNCs for 1-5 d, cells viability showed a great increase and no significant difference was observed compared with tissue culture plate (TCP) control, indicating their high biocompatibility (Figure 5A). Further Live-Dead staining fluorescent images exhibited that cells showed good attachment morphology after incubated with BGNCs at different concentrations and no dead cells were observed after $1 \mathrm{~d}$ (Figure 5B-D). These results were consistent with previous findings that BGbased bioactive materials have a good cell biocompatibility especially the osteoblasts. ${ }^{[31]}$

BMSCs were used as model cells to investigate the miRNA transfection efficiency of BGNCs. After transfected for $48 \mathrm{~h}$, the significant green fluorescence of miRNA in the cells was
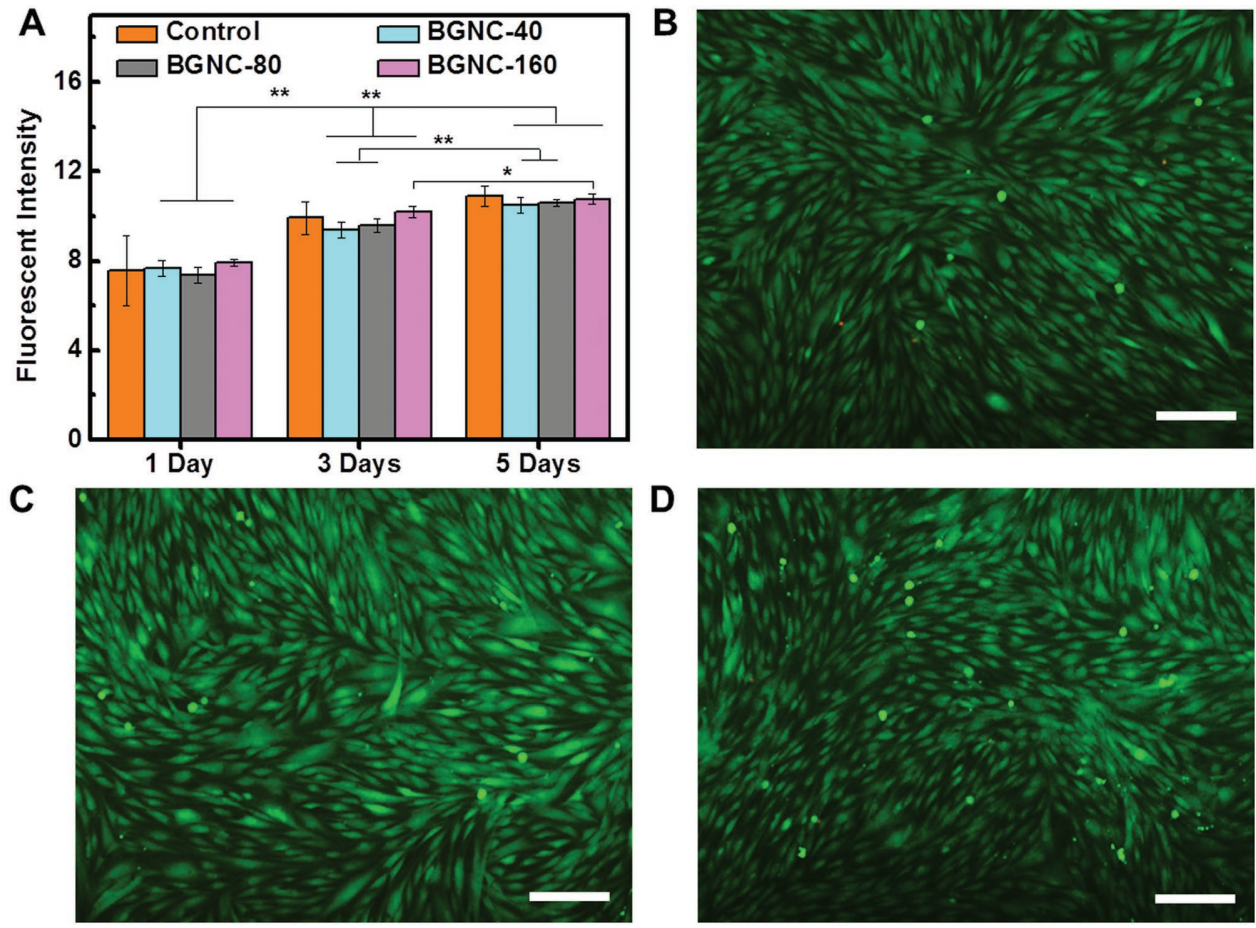

Figure 5. Cellular biocompatibility evaluation. A) BMSCs viability and proliferation of cells after treated with BGNCs at concentrations of 40 , 80 , and $160 \mathrm{mg} \mathrm{mL}^{-1}\left(* p<0.05\right.$ and ${ }^{*} * p<0.01, n=5$ ). B-D) Live-Dead staining fluorescence images of cells after culture with BGNCs for $1 \mathrm{~d}$ at B) $40 \mathrm{mg} \mathrm{mL}{ }^{-1}$, C) $80 \mathrm{mg} \mathrm{mL}^{-1}$, and D) $160 \mathrm{mg} \mathrm{mL}^{-1}$ (scale bar: $200 \mu \mathrm{m}$ ). 

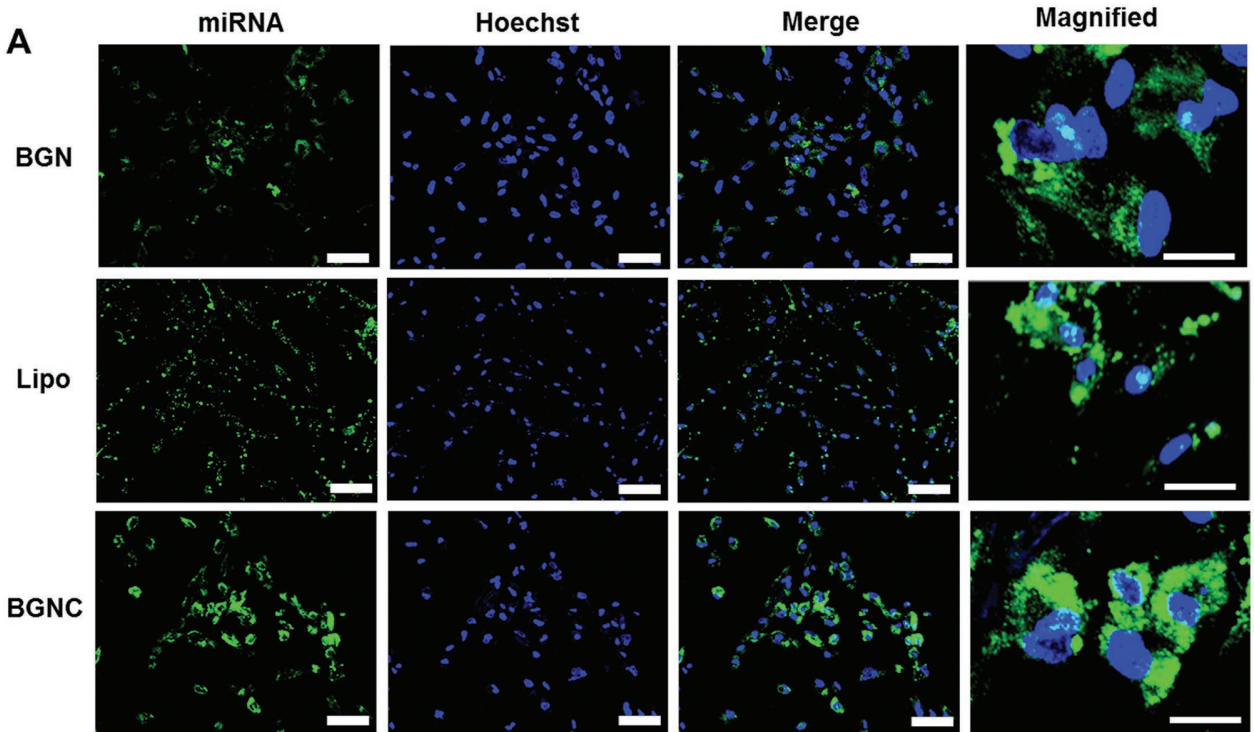

B
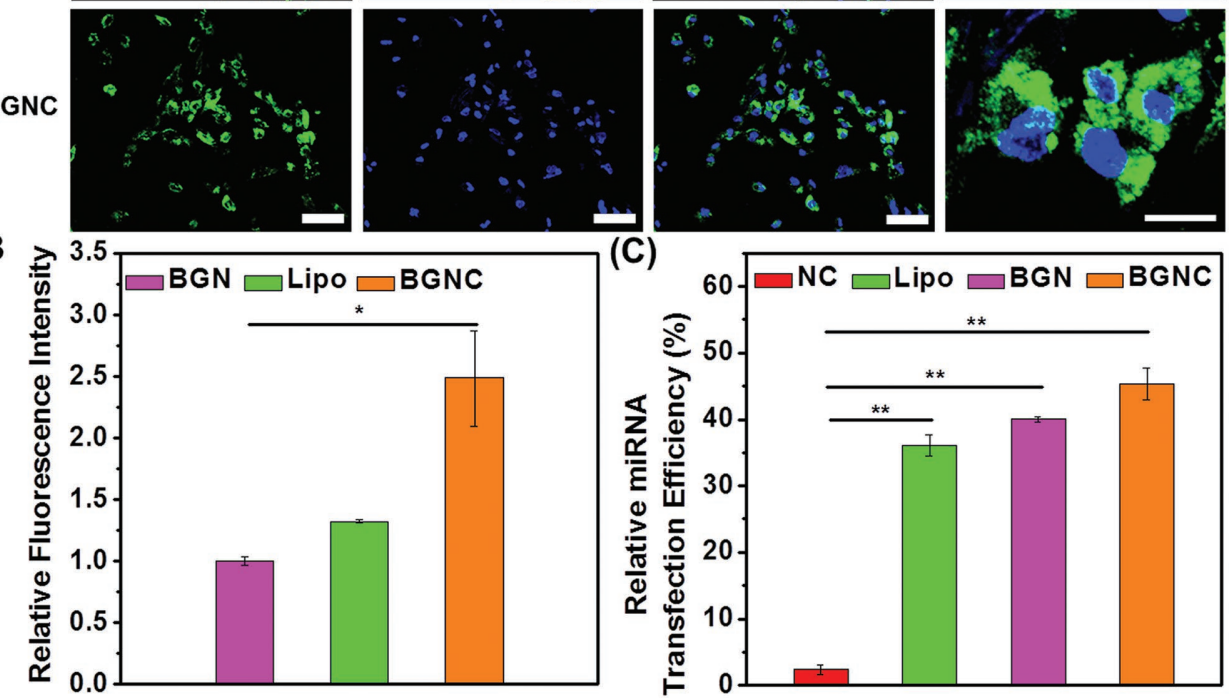

Figure 6. MiRNA transfection efficiency evaluation of BGNCs. A) CLSM images of BMSCs after transfected with BGNC@miRNA for 48 h, using BGN and Lipo as controls (scale bar: $100 \mu \mathrm{m}$ ). The miRNA was labeled as green by FAM and cell nuclear was stained as blue by Hoechst. B) Quantitative fluorescent intensity analysis of cells based on CLSM images after transfected by various miRNA complexes. C) Flow cytometry analysis of cells after transfected by various miRNA complexes, demonstrating the miRNA transfection efficiency $(* p<0.05$, ** $p<0.01, n=5)$.

observed clearly in BGNCs group compared with Lipo/BGN group, suggesting the high efficient miRNA intracellular delivery of BGNCs (Figure 6A). The magnified confocal scanning microscope (CLSM) images of cells showed that miRNA was mainly distributed around the cell nucleus, implying that miRNA was successfully transported into the cells. Moreover, quantitative analysis based on CLSM images showed that the mean fluorescent intensity of BGNCs group was significantly higher than that of Lipo and BGNs group (Figure 6B). The flow cytometry examination further demonstrated the difference of miRNA transfection efficiency for various groups (Figure 6C). Almost negligent transfection ability $(2.3 \%)$ in BMSCs was found in naked FAM-labeled miRNA group (NC). BGNCs group demonstrated significantly high miRNA transfection efficiency $(45.3 \%)$, as compared to BGNs group $(40.1 \%)$ and commercial Lipo group (35.1\%). The excellent miRNA delivery and transfection efficiency of BGNCs should be attributed to their ultralarge pore structure and ultrahigh miRNA loading efficiency, as well as strong miRNA protection capacity.

In this study, the used miRNA-5106 (miR-5106) has been shown to have positive osteogenic inductive ability through upregulating several osteogenic genes expressions. ${ }^{[32]}$ Here, we investigate the effect of BGNC-based miR-5106 delivery on osteogenic differentiation markers expressions (Runx2, Opn, and $A l p$ ) in BMSCs by qRT-PCR analysis (Figure 7). As compared to pure BGNs and BGNCs group, BGN@miRNA and BGNC@miRNA group showed the significantly high Runx2, Opn, and Alp expression level during the culture periods of 7 and $14 \mathrm{~d}$, which suggested that BGNS and BGNCs could efficiently deliver miR-5106 to enhance osteogenic differentiation of cells. Additionally, Runx2, Opn, and Alp expression increased gradually in all groups during $14 \mathrm{~d}$. At day 14 , the Runx2, Opn, and Alp expression in BGNC@miRNA group was 1.5 times, 2.0 times, and 1.8 times higher than those in BGN@miRNA group. These results further indicated that BGNCs could efficiently deliver miRNA and enhance the osteogenic differentiation of BMSCs, and may have a great potential for in vivo bone regeneration as a new gene-activated bioactive materials.

\subsection{In Vivo Bone Tissue Regeneration}

The promising in vitro results of BGNCs, including miRNA transfection and osteogenic differentiation, encouraged us 
A

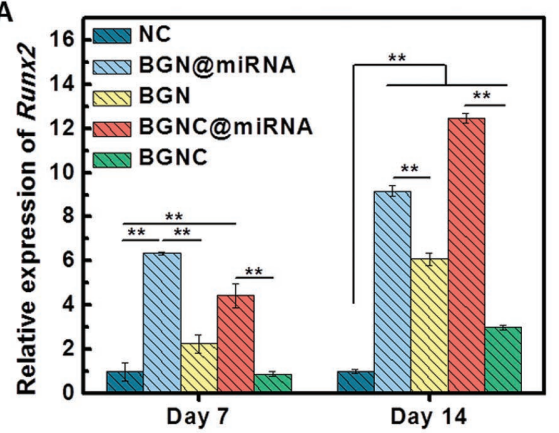

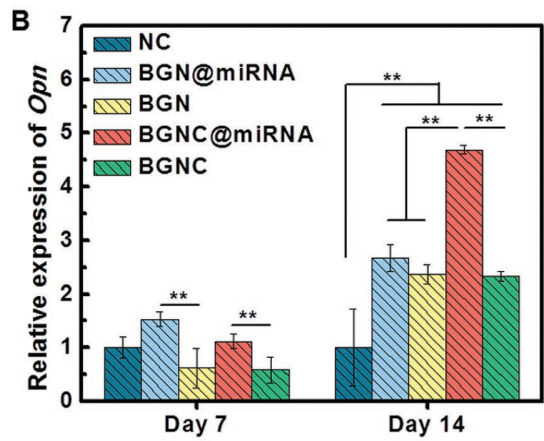

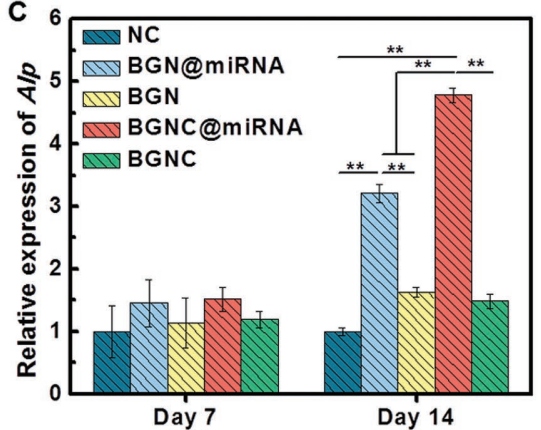

Figure 7. Osteogenic gene maker expressions of cells after induced for 7 and $14 \mathrm{~d}$ by various miRNA-5106 complexes. A) Runx2; B) Opn; C) Alp. Significantly enhanced Runx2, Opn, Alp expressions were observed at day 14 in BGNCs group, as compare to other groups. The tissue culture plate (TCP) with naked miRNA-5106 was used as negative control (NC) $(* p<0.05, * * p<0.01, n=3)$.

to investigate the effect of BGNCs on in vivo bone repair and regeneration. The bone regeneration ability of BGNCs and BGNC@miRNA was investigated by a rat critical sized cranial defect model. After implantation in defect site for four weeks, the bone tissue samples were removed and were scanned by an X-ray microCT systems to evaluate the newly formed bone tissue. As shown in Figure 8A, blank NC group shows little new bone formation near the defects border, but newly formed bone tissue could be clearly observed in BGNCs and BGNs group. In addition, large amount of new bone formation could be found after treated by BGNC@miRNA group, which was significantly higher than BGNC/BGN/BGN@miRNA group. Further quantitative bone tissue analysis exhibited that BGNC@miRNA group demonstrated a significantly high new bone volume and trabecular thickness, compared with BGNCs, BGN@miRNA, and BGNs group (Figure 8B,C).

The newly formed bone tissues were further examined through hematoxylin and eosin (H\&E) and Masson's trichrome staining (Figure 9). H\&E staining showed the results of inflammation response and new bone formation (NB in red). All groups exhibited the low inflammation response in defect site. There was minimal new bone formation in NC
A

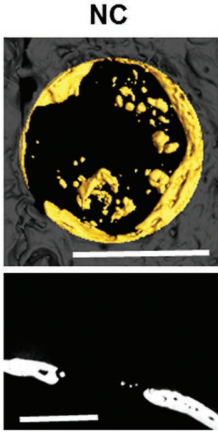

B

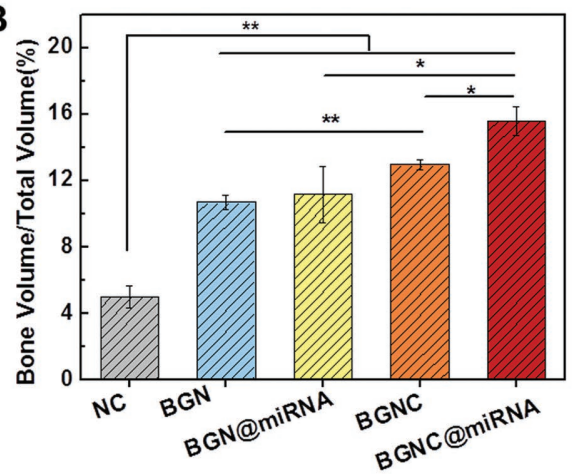

BGN

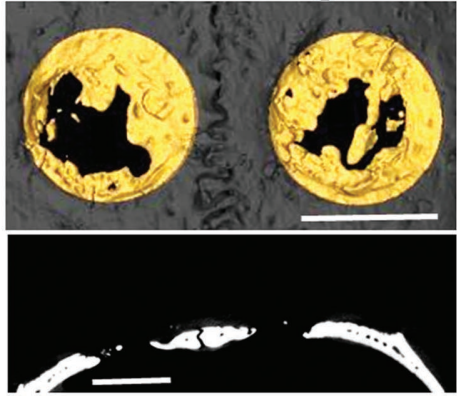

C

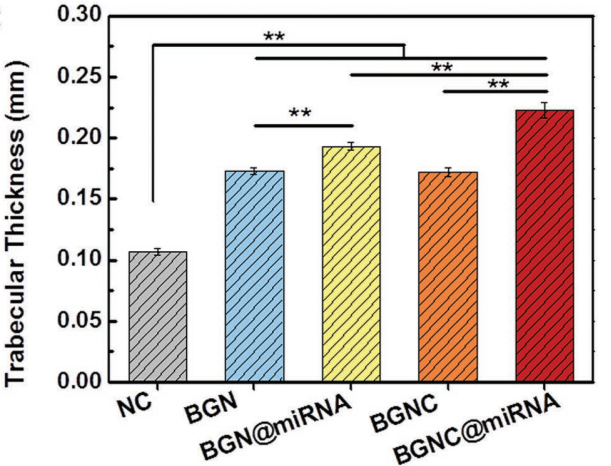

Figure 8. In vivo bone tissue regeneration of BGNs, BGNCs,BGN@miRNA, BGNC@miRNA after implantation in critical-sized defect for four weeks. A) MicroCT images showing the repair and reconstruction of frontage (up) and cross-section (down) in cranial defects (Scale bars $=5 \mathrm{~mm}$ ). $\mathrm{B}, \mathrm{C})$ Quantitative analysis of new bone volume fraction B) and trabecular thickness C) calculated from the micro-CT images (* $p<0.05$, *** $p<0.01, n=3)$. Significantly high new bone formation was observed in BGNC@miRNA group. 

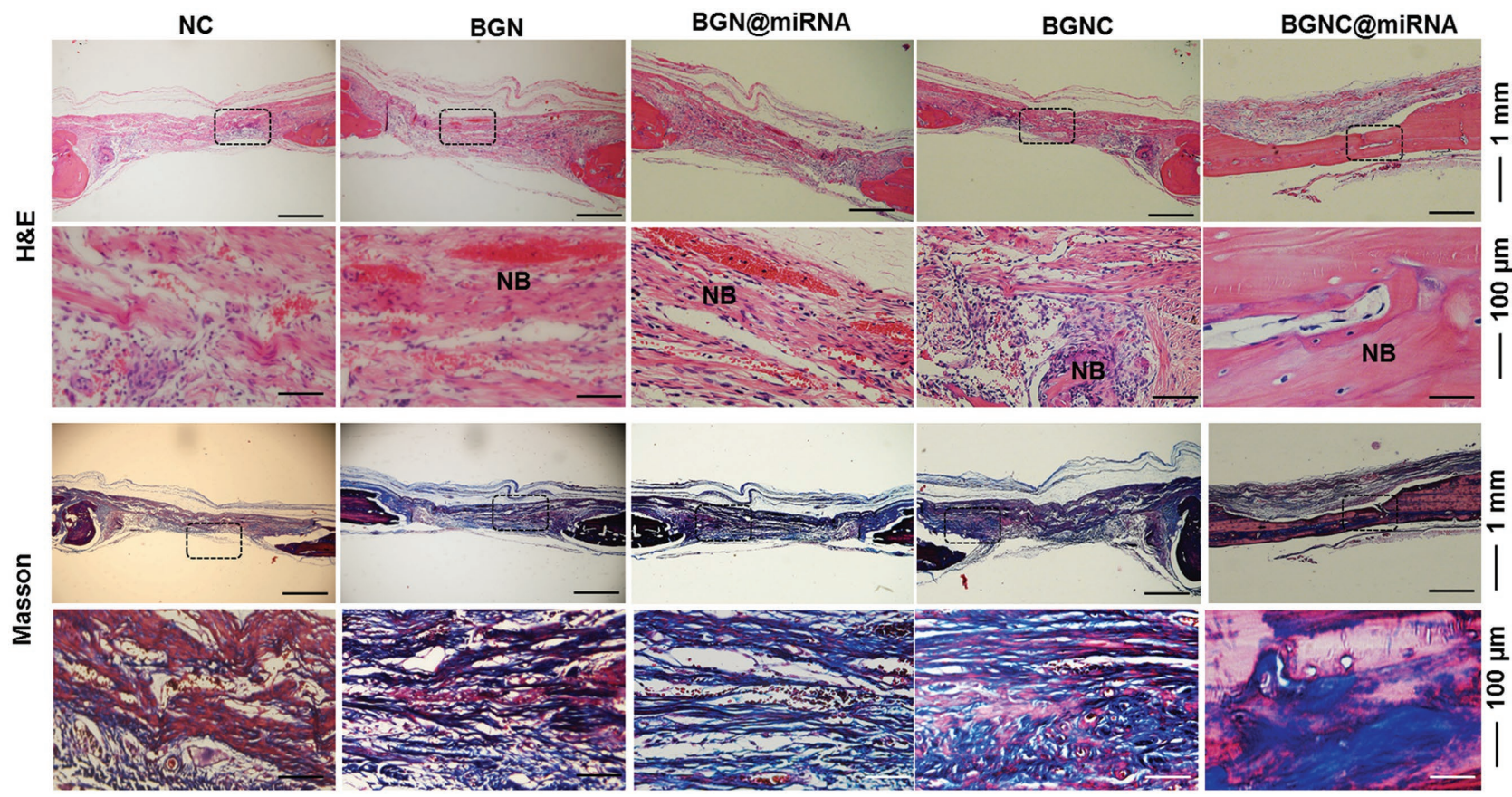

Figure 9. Histological analysis of new bone formation after $4 \mathrm{w}$ implantation in defect site. H\&E staining demonstrates the new bone formation (NB) dependent on various samples; Masson staining exhibits the collagen deposition (dark blue) in newly formed bone tissue. BGN@miRNA, BGNC, and BGNC@miRNA group significantly enhanced bone formation and collagen deposition in vivo.

group and significantly increased bone tissue was observed in BGN-based groups.BGNC@miRNA group induced the best bone formation after four weeks implantation in defect site. Masson trichrome staining was used to identify the collagen deposition in bone defect site after repair. Significantly high and dense collagen deposition (dark site) could be found in BGNCs and BGNC@miRNA group. Limited positive staining in NC group suggested their low bone collagen deposition. The histological results were in accordance with those of microCT, demonstrating the rapid bone regeneration ability of BGNC@ miRNA.

In bone tissue regeneration, conventional bioactive materials including bioactive glass and calcium phosphate ceramics-based nanocomposites have shown promising results in enhancing bone tissue formation. ${ }^{[33,34]}$ The geneactivated bioactive materials with intrinsic gene loading capacity are less demonstrated, although they are rather important for tissue regeneration and disease therapy. In this paper, the developed BGNCs exhibited ultrahigh miRNA loading and excellent delivery performance for enhanced osteogenic differentiation and bone formation. Inorganic nanoparticles with ultralarge pores have shown special advantages in drug and gene delivery applications. ${ }^{[35]}$ As novel nonviral gene vectors, compared with silica nanoparticles, BGNs present inherent gene binding ability through their unique metal-organic complex mechanism. ${ }^{[20]}$ Unfortunately, the gene may be only loaded on the surface in BGNs due to their small pore structure (below $5 \mathrm{~nm}$ ). Herein, by using branched PEI as a template, BGNCs with a monodispersed size, and ultralarge porous structure were successfully fabricated. Then, the ultrahigh miRNA loading efficiency, signifi- cantly enhanced osteogenic genes expressions, and bone formation were achieved through BGNCs-mediated delivery. As compared to conventional nonviral vectors such as liposomes and cationic polymers, BGNCs possess several advantages. First, large-scale fabrication of BGNCs could be achieved facilely, which is very important for wide clinical applications. Second, BGNCs show high biocompatibility and intrinsic osteogenic activity, which may have synergetic effect with miRNA delivery in bone formation. Based on these excellent properties, BGNCs may have great potential as next-generation nonviral vector to deliver genes for tissue regeneration and disease therapy.

\section{Conclusion}

In summary, we developed a novel and simple strategy for large-scale synthesis of monodispersed BGNCs with ultralarge mesopores $(\approx 20 \mathrm{~nm})$ and tailored nanosize $(100$ $500 \mathrm{~nm})$ using branched PEI as the template and catalyst. BGNCs show an excellent apatite-forming ability due to their high surface area and pore structure. BGNCs can support and enhance BMSC attachment and proliferation, indicating their good cellular compatibility. Significantly, BGNCs demonstrate ultrahigh miRNA loading efficiency which is 19 times higher than conventional BGNs. BGNCs can protect, deliver miR-5106 to enhance the osteogenic differentiation of BMSCs and significantly promote the new bone formation in critical sized cranial defects in rats. Therefore, based on the excellent pore structure, unique gene binding ability and biosafty, BGNCs would be a favorable vehicle to deliver various genes 
for enhancing tissue regeneration and cancer therapy. As a new-generation gene vector, further in vivo tissue regeneration and cancer therapy applications based on BGNCs should be demonstrated.

\section{Experimental Section}

Materials: PEI ( $\mathrm{Mn}$ 25000), tetraethyl orthosilicate (TEOS), triethylphosphate (TEP), calcium nitrate tetrahydrate (CN), ethyl alcohol (99\% ETOH), tris, agarose, boric acid were purchased from Sigma-Aldrich. Opti-MEM, AlamarBlue cell viability kit, LIVE-DEAD mammalian cell viability stain kit, Hoechst 33342 and 4-(2-hydroxyethyl)1-piperazine- $N^{\prime}$-2-ethanesulfonic acid (HEPES) were obtained from Thermo Fisher. Normal miRNA and carboxyfluorescein (FAM) labeled miRNA (miR-5106) were bought from Genepharma (China). Deionized water (DW) was used for all experiments. All chemicals were used as received without further purification.

Synthesis and Characterizations of Monodispersed BGNCs: Monodispersed BGNCs with ultralarge pores were fabricated via a sol-gel method using PEI as the catalyst and structure-directing agent. Briefly, PEI was dissolved in $500 \mathrm{~mL}$ of ethyl alcohol/water solution $(3 / 1=v / v)$ under magnetically stirring, followed by addition of $10 \mathrm{~mL}$ TEOS, and the resulting mixture was stirred for $30 \mathrm{~min}$. Then TEP and $\mathrm{CN}$ were added to the reaction system in sequence and the reaction was kept at $40{ }^{\circ} \mathrm{C}$ for $3 \mathrm{~h}$. The resulted product was collected by centrifugation and purification by washing with ETOH/DW for several times. The nanoparticle size of BGNCs was controlled through adding different amount of PEI. Specifically, the mass ratio of TEOS to PEI were $1: 0.06,1: 0.1,1: 0.2$, and 1:0.3, and the final products were denoted as $6 \%$ BGNCs, $10 \%$ BGNCs, $20 \%$ BGNCs, and $30 \%$ BGNCs. Additionally, conventional BGNs with the same chemical composition as BGNCs were prepared according to the previous report and used as a control material in this study. ${ }^{[36]}$

The nanostructure and morphology of BGNCs were observed by a TEM (HT-7700, Hitachi). The phase, chemical structure and composition of BGNCs were investigated by XRD (DMAX/A, Rigaku), FTIR spectroscopy (Nicolet 6700, Thermo Sci.) and X-ray EDS (Quanta 250 FEG, FEI). Nitrogen adsorption-desorption measurement and BET method was carried out to analyze the specific surface area and pore size distributions of BGNCs (Quadrasorb SI-3, Quantachrome). UVvis absorption spectra were recorded on a UV-vis spectrophotometer (Lambda 35, PerkinElmer) at room temperature.

Apatite-Forming Bioactivity Evaluation of BGNCs: The apatite-forming investigation of BGNCs was performed in simulated body fluids (SBF) for $3 \mathrm{~d}$, according to the previous report. ${ }^{[36]}$ In a typical process, BGNCs was soaked into SBF at a concentration of $10 \mathrm{mg} \mathrm{mL}^{-1}$ and shaken at $100 \mathrm{rpm}$ at $37{ }^{\circ} \mathrm{C}$ for predetermined times. The morphology and structures of apatite formed on the surface of BGNC was observed by TEM, and further analyzed by FTIR and XRD.

Formation and Stability Analysis of BGNC@miRNA Complexes: Based on the optimum monodispersed size and controlled morphology, $10 \%$ BGNCs were used in further miRNA loading, delivery, and biocompatibility studies. The miRNA (miR-5106 from GenePharma, Shanghai) loading capacity and stability of BGNCs was analyzed using a gel retardation assay, ${ }^{[37]} \mathrm{BGNs}$ were used as a control. Briefly, the BGNC@miRNA orBGN@miRNA complexes at different mass ratios ranging from 0:1 to 64:1 were prepared as the following procedure. Different amount of BGNCs/BGNs were mixed with $13 \mu \mathrm{g} \mathrm{mL}$ miRNA in HEPES and incubated for $30 \mathrm{~min}$ at $37^{\circ} \mathrm{C}$. Then the resulting complexes were loaded on $2 \%$ agarose gel to conduct the gel retardation assay (110 V for $15 \mathrm{~min}$ ) on an electrophoresis system (HE120, Tanon). The mean intensity of miRNA bands and pictures-taking were investigated by a gel imaging system (Gel Doc XR+, Bio-rad). The binding percentage was calculated as the following equation

$B p=(D a-D b) /(D c-D b) \times 100 \%$ where $B p$ is the miRNA binding percentage, $D a$ is the densities of miRNA bands in the wells with BGNC/BGN, Dc represents the densities of miRNA bands in the wells without $B G N C / B G N$, and $D b$ is the density of background, respectively.

The stability investigation of BGNC@miRNA complexes was performed in the presence of serum, using BGNs and commercial Lipofectamine 3000 (Lipo) as the controls. Briefly, BGNC@miRNA complexes solutions were added with $25 \%$ fetal bovine serum (FBS) and incubated for predetermined times $(0-24 \mathrm{~h})$. Afterward, ribonuclease inhibitor (RRI, Tiangen) was added to the solutions to inhibit the further degradation of miRNA. Then the samples were collected and treated with heparin and subsequently loaded on $2 \%$ agarose gel for gel retardation assay, the mean intensity of miRNA bands were analyzed by the gel imaging system.

Cytotoxicity Investigation: The cellular biocompatibility of BCNCs was carried out by using the BMSCs (from Cell Bank, Chinese Academy of Sciences, China), according to previous report. ${ }^{[32]}$ The live cellular morphology and proliferation was evaluated by using a Live-Dead kit (Life Science) and alamar blue assay kit (Life Science), respectively. Briefly, cells were seeded in a 96 well plate at a density of 4000 cells per well and incubated in a growth medium (DMEM, 10\% FBS, Life Sciences) in a humidified incubator at $37{ }^{\circ} \mathrm{C}$ with $5 \% \mathrm{CO}_{2}$. After attachment for $24 \mathrm{~h}$, the medium was removed, followed by adding the growth medium containing various BGNCs concentrations $\left(40-160 \mu \mathrm{g} \mathrm{mL}^{-1}\right)$. The cells were cultured with $\mathrm{BGNCs}$ for $1,3,5 \mathrm{~d}$. At the predetermined time point, the cells were washed by phosphate buffered saline (PBS) for three times and incubated in $100 \mu \mathrm{L}$ of Live-Dead solution for $45 \mathrm{~min}$ at $37^{\circ} \mathrm{C}$. The live cell morphology was observed via an inverted fluorescent microscope (Olympus, IX53). After 1, 3, and $5 \mathrm{~d}$ incubation, the live cell viability was measured by using an Alamar Blue kit in accordance with the manufacture's instruction. The live cell fluorescence was measured at an excitation/emission wavelength of $530 / 600 \mathrm{~nm}$ on a microreader (SpectraMax, Molecular Services). TCP without materials was used as a control. At least five species per sample was tested.

Transfection Efficiency Investigations: For demonstrating the delivery efficiency of miRNA by BGNCs, the cellular uptake and intracellular fluorescence expression of BGNC@miRNA complexes were evaluated by laser CLSM (FV1200, Olympus) and a flow cytometer (CytoFLEX Coulter Beckman), using BGNs and Lipo as controls. For CLSM observation, cells were seeded on glass slides at a density of 5000 cells per well in a growth medium. After $24 \mathrm{~h}$, cells were rinsed by PBS and incubated with the fluorescent labeled miRNA complexes (BGNC@ miRNA,BGN@miRNA, or Lipo@miRNA) in a transfection medium. After culture for $24 \mathrm{~h}$, the medium was removed and cells were washed by PBS for three times. The cell nucleus was stained as blue by Hoechst (Life Technologies). Then the transfected cell images were observed under the CLSM.

Flow cytometer (CytoFLEX, Coulter Beckman) was used to evaluate the cellular uptake of miRNA-loaded $10 \%$ BGNC/BGN/Lipo. Typically, BMSCs transfected with miRNA-loaded 10\%BGNC/BCN/Lipo were washed by Dulbecco's phosphate buffered saline (Thermo Fisher Scientific) for two times, after trypsinization and centrifugation process, the cells were finally suspended in $100 \mu \mathrm{L}$ PBS supplemented with $2 \%$ FBS and $100 \mu \mathrm{L}$ of $4 \%$ paraformalclehyde. After incubated for $2 \mathrm{~h}$ at $4{ }^{\circ} \mathrm{C}$, the percentage of cells successfully transfected were analyzed by detecting the fluorescence intensity of samples through flow cytometry and analyzed by CytExpert software (Beckman Coulter).

Osteogenic Genes Expressions Examination: The BMSCs were transfected with different miRNA complexes and cultured for different times ( 7 and $14 \mathrm{~d}$ ) for evaluating osteogenic genes expressions ( $A / p$, Runx2, and $O p n)$ using a quantitative real-time polymerase chain reaction (qRT-PCR, Bio-rad). The $\alpha$-MEM (Sigma) supplemented with $10 \%$ FBS (Gibco), $50 \mathrm{mg} \mathrm{mL}^{-1}$ ascorbic acid, $10 \times 10^{-3} \mathrm{M}$ $\alpha$-glycerophosphate (Sigma) and $10 \times 10^{-9} \mathrm{M}$ dexamethasone (Sigma) was used as osteogenic inductive medium. $10 \%$ BGNCs were used to load miRNA for osteogenic differentiation experiment. Typically, cells were seeded in a 24 well plate at a density of $2 \times 10^{4}$ cells per well and cultured in DMEM supplemented with $10 \%$ FBS $(\mathrm{v} / \mathrm{v})$ for 
$24 \mathrm{~h}$. Then the cells were rinsed by PBS and treated with different samples (BGNC@miRNA,BGN@miRNA,BGNC, and BGN). After being incubated for $24 \mathrm{~h}$, the medium were changed to osteogenic inductive medium. The medium were replaced by fresh medium every $3 \mathrm{~d}$. At every specific time point, cells were collected and total RNA were extracted using Tripure (Roche), then single-stranded cDNA was synthesized using a RevertAid First Strand cDNA Synthesis kit (Roche) according to manufacturer's instructions. qRT-PCR was carried out by employing iTaq universal SYBR Green supermix (Applied Biosystems 7500, Bio-rad) with the gene-specific primers listed in Table S1 (Supporting Information). The qRT-PCR amplifications were conducted under $95{ }^{\circ} \mathrm{C}$ for $5 \mathrm{~min}, 40$ cycles of $95^{\circ} \mathrm{C}$ for $10 \mathrm{~s}, 60^{\circ} \mathrm{C}$ for $30 \mathrm{~s}$, and $70{ }^{\circ} \mathrm{C}$ for $10 \mathrm{~min}$.

Critical-Sized Bone Regeneration Evaluation In Vivo: The in vivo bone regeneration ability of BGNCs and BGNC@miRNA were investigated by a rat cranial critical sized defect model $(5 \mathrm{~mm}$ in diameter, Sprague Dawley rats, six weeks, male, 200-220 g). Animal surgeries and euthanasia work was conducted in compliance with the animal care and use guidelines of National Institutes of Health and the animal research committee of Xi'an Jiaotong University. Briefly, the rats were narcotized with $10 \mathrm{wt} \%$ chloral hydrate under a rigorous aseptic condition $(n=$ 6 per group), $5 \mathrm{~mm}$ diameter defects were made at the both sides of calvarium. Before implantation, samples were mixed with biomedical fibrin glue (Porcine Fibrin Sealant Kit, Hangzhou Puji Pharmaceutical Technology) at a weight ratio of 1:1. The as-prepared hybrid solution could be formed to hydrogel in defect site once implantation. The critical sized defect was filled with fibrin glue hydrogel containing BGN, BGN@ miRNA, BGNC,BGNC@miRNA, using pure fibrin glue hydrogel as a negative control (NC). After implantation for four weeks, the rats were sacrificed, and the implanted materials/surrounding native bones were dissected and fixed in 10\% neutral buffered formalin for microCT analysis and histological evaluation. MicroCT analysis was performed on a 3D microfocus X-ray microcomputed tomography system (Y.CHEETAH*, YXLON) to quantitatively analyze the newly formed bone within the defects. For the histology evaluation, bone samples were decalcified in 10\% EDTA and embedded in paraffin. Then the samples were sectioned (5 $\mu \mathrm{m}$ thick) and stained with H\&E, Sigma and Masson's trichrome (Sigma), respectively. The bone volume and trabecular thickness was calculated based on the microCT analysis. At least three rats per group were evaluated.

Statistical Analysis: All results were expressed as mean value \pm standard deviation. SPSS Statistics software (version 13.0) was utilized for all the statistical analysis, and Student's $t$-test was carried out to evaluate the statistically significant differences between two groups. It was considered that the differences were significant when $* p$ values < 0.05 or $* * p<0.01$. All experiments were performed at least in triplicate.

\section{Supporting Information}

Supporting Information is available from the Wiley Online Library or from the author.

\section{Acknowledgements}

The authors acknowledge the valuable comments of potential reviewers. This work was supported by the State Key Laboratory for Mechanical Behavior of Materials (No. 20161801), the Fundamental Research Funds for the Central Universities (No. XJJ2014090), and National Natural Science Foundation of China (Nos. 51502237 and U1501245).

\section{Conflict of Interest}

The authors declare no conflict of interest.

\section{Keywords}

bioactive glass, bone regeneration, miRNA delivery, nanoclusters, silicabased biomaterials

Received: May 18, 2017

Revised: June 18, 2017

Published online: July 24, 2017

[1] G. A. Rodan, T. J. Martin, Science 2000, 289, 1508.

[2] C.-H. Lu, Y.-H. Chang, S.-Y. Lin, K.-C. Li, Y.-C. Hu, Biotechnol. Adv. 2013, 31, 1695.

[3] B. Peng, Y. Chen, K. W. Leong, Adv. Drug Delivery Rev. 2015, 88, 108.

[4] L. Zheng, Q. Tu, S. Meng, L. Zhang, L. Yu, J. Song, Y. Hu, L. Sui, J. Zhang, M. Dard, J. Cell. Physiol. 2017, 232, 182.

[5] M. Sriram, R. Sainitya, V. Kalyanaraman, S. Dhivya, N. Selvamurugan, Int. J. Biol. Macromol. 2015, 74, 404.

[6] N.-F. Sun, Z.-A. Liu, W.-B. Huang, A.-L. Tian, S.-Y. Hu, Crit. Rev. Oncol. Hematol. 2014, 89, 352.

[7] H. Wang, Y. Jiang, H. Peng, Y. Chen, P. Zhu, Y. Huang, Adv. Drug Delivery Rev. 2015, 81, 142.

[8] H. Yin, R. L. Kanasty, A. A. Eltoukhy, A. J. Vegas, J. R. Dorkin, D. G. Anderson, Nat. Rev. Genet. 2014, 15, 541.

[9] A. Ganju, S. Khan, B. B. Hafeez, S. W. Behrman, M. M. Yallapu, S. C. Chauhan, M. Jaggi, Drug Discovery Today 2017, 22, 424.

[10] M. Ramamoorth, A. Narvekar, J. Clin. Diagn. Res. 2015, 9, GE01.

[11] F. Baino, S. Fiorilli, C. Vitale-Brovarone, Acta Biomater. 2016, 42, 18.

[12] A. L. Maçon, T. B. Kim, E. M. Valliant, K. Goetschius, R. K. Brow, D. E. Day, A. Hoppe, A. R. Boccaccini, I. Y. Kim, C. Ohtsuki, J. Mater. Sci.: Mater. Med. 2015, 26, 1.

[13] B. Lei, L. Wang, X. Chen, S. K. Chae, J. Mater. Chem. B. 2013, 1, 5153.

[14] L. L. Hench, N. Roki, M. B. Fenn, J. Mol. Struct. 2014, 1073, 24.

[15] B. Lei, X. Chen, Y. Wang, N. Zhao, C. Du, L. Fang, Biomed. Mater. 2010, 5, 054103

[16] L. L. Hench, J. M. Polak, Science 2002, 295, 1014.

[17] A. El-Fiqi, T.-H. Kim, M. Kim, M. Eltohamy, J.-E. Won, E.-J. Lee, H.-W. Kim, Nanoscale 2012, 4, 7475.

[18] X. Li, X. Chen, G. Miao, H. Liu, C. Mao, G. Yuan, Q. Liang, X. Shen, C. Ning, X. Fu, J. Mater. Chem. B 2014, 2, 7045.

[19] F. Chen, G. Wang, J. I. Griffin, B. Brenneman, N. K. Banda, V. M. Holers, D. S. Backos, L. Wu, S. M. Moghimi, D. Simberg, Nat. Nanotechnol. 2016, 12, 387.

[20] M. Yu, Y. Xue, P. X. Ma, C. Mao, B. Lei, ACS Appl. Mater. Interfaces 2017, 9, 8460

[21] C. Vichery, J.-M. Nedelec, Materials 2016, 9, 288.

[22] J. Wei, Z. Sun, W. Luo, Y. Li, A. A. Elzatahry, A. M. Al-Enizi, Y. Deng, D. Zhao, J. Am. Chem. Soc. 2017, 139, 1706.

[23] X. Guan, Z. Guo, T. Wang, L. Lin, J. Chen, H. Tian, X. Chen, Biomacromolecules 2017, 18, 1342.

[24] Q. Hu, Y. Li, G. Miao, N. Zhao, X. Chen, RSC Adv. 2014, 4, 22678.

[25] L. L. Hench, H. Paschall, J. Biomed. Mater. Res., Part A 1973, 7, 25.

[26] T. Kokubo, H.-M. Kim, M. Kawashita, Biomaterials 2003, 24, 2161.

[27] A. L. Macon, T. B. Kim, E. M. Valliant, K. Goetschius, R. K. Brow, D. E. Day, A. Hoppe, A. R. Boccaccini, I. Y. Kim, C. Ohtsuki, T. Kokubo, A. Osaka, M. Vallet-Regi, D. Arcos, L. Fraile, A. J. Salinas, A. V. Teixeira, Y. Vueva, R. M. Almeida, M. Miola, C. Vitale-Brovarone, E. Verne, W. Holand, J. R. Jones, J. Mater. Sci.: Mater. Med. 2015, 26, 115.

[28] S. J. Quinn, A. R. Thomsen, J. L. Pang, L. Kantham, H. BraunerOsborne, M. Pollak, D. Goltzman, E. M. Brown, Am. J. Physiol. 2013, 304, E310. 
[29] J. Tu, A. L. Boyle, H. Friedrich, P. H. Bomans, J. Bussmann, N. A. Sommerdijk, W. Jiskoot, A. Kros, ACS Appl. Mater. Interfaces 2016, 8, 32211

[30] M. Wu, Q. Meng, Y. Chen, L. Zhang, M. Li, X. Cai, Y. Li, P. Yu, L. Zhang, J. Shi, Adv. Mater. 2016, 28, 1963.

[31] G. Kaur, S. G. Waldrop, V. Kumar, O. P. Pandey, N. Sriranganathan, Biocompatible Glasses 2016, 53, 19.

[32] M. Yu, B. Lei, C. Gao, J. Yan, P. X. Ma, Nano Res. 2017, 10 49.
[33] M. N. Rahaman, D. E. Day, B. S. Bal, Q. Fu, S. Jung, L. Bonewald, A. Tomsia, Acta Biomater. 2011, 7, 2355.

[34] M. Mastrogiacomo, S. Scaglione, R. Martinetti, L. Dolcini, F. Beltrame, R. Cancedda, R. Quarto, Biomaterials 2006, 27, 3230.

[35] D. Niu, Z. Liu, Y. Li, X. Luo, J. Zhang, J. Gong, J. Shi, Adv. Mater. 2014, 26, 4947.

[36] Y. Xue, Y. Du, J. Yan, Z. Liu, P. X. Ma, X. Chen, B. Lei, J. Mater. Chem. B 2015, 3, 3831

[37] X. Du, B. Shi, Y. Tang, S. Dai, S. Z. Qiao, Biomaterials 2014, 35, 5580. 